\title{
On the holographic basis of loop quantum cosmology
}

\author{
C. A. S. Silva ${ }^{\text {a }}$ \\ Instituto Federal de Educação Ciência e Tecnologia da Paraíba (IFPB), Campus Campina Grande, Rua Tranquilino Coelho Lemos, 671, \\ Jardim Dinamérica I., Campina Grande, Brazil
}

Received: 21 November 2017 / Accepted: 10 May 2018 / Published online: 24 May 2018

(C) The Author(s) 2018

\begin{abstract}
In this work, we obtain the loop quantum cosmology dynamical equations, plus a positive cosmological constant, from the Bekenstein-Hawking entropy-area relation given by Loop Quantum Black Holes, by the use of the Jacobson formalism (Phys Rev Lett 75:1260, 1995). The results found out may set a still absent connection between holography and the description of the cosmos given by loop quantum cosmology.
\end{abstract}

\section{Introduction}

Loop quantum gravity (LQG) proposes a way to model the behavior of spacetime in situations where its atomic characteristic arises [2-6]. Among these situations, the nature of our universe near the Big Bang singularity is described by loop quantum cosmology (LQC) [7]. This description of cosmology which takes into account effects of quantum gravity has become very popular during the last decade, because it allows making contact with the observational activity $[8,9]$.

The main result in LQC is the resolution of the Big Bang singularity, since there are long standing prospects that the General Relativity initial singularity must be solved in the context of a quantum gravity theory. In the case of LQC, the Big Bang singularity is naturally replaced by a bounce when the curvature becomes stronger at the Planckian regime. At this point, the universe density does not become infinite anymore, but assumes a maximum finite critical value. A quantum bridge forms in the place of the initial singularity and the universe can tunnel through it. In this sense, the quantum evolution of the universe extends through the Big Bang. Such results open the possibility that our universe could have its origin in a prior contraction phase [10]. The possibility of a phase(s) for the universe before the hot Big Bang, on the other hand, raises important questions related with the thermodynamics of our universe. Among these questions, we have the

a e-mail: carlos.souza@ifpb.edu.br problem about the origin of the cosmological entropy and the arrow of time [11]. In order to address these issues, its is necessary to answer a question that remains open until now: what is the correct way to count the spacetime states in agreement with the LQC description of the universe?

On the other hand, among the results coming from black hole thermodynamics, we have the Bekenstein-Hawking formula, where the entropy of a black hole is given as proportional to its horizon area: $S=A / 4 \hbar G$ [12]. Behind the simplicity of this expression, lies a deep intersection between two theories that remain at odds until now, gravity and quantum mechanics. Interestingly, Bekenstein-Hawking formula is one of the few places in physics where the Newton's gravitational constant $G$ meets the Planck constant $\hbar$. Moreover, the Bekenstein-Hawking formula consists in the basis of the holographic principle which sets how many degrees of freedom there are in nature at the most fundamental level [13-16].

Consequently, one may wonder that, the way to discuss the universe thermodynamical evolution in the context of LQC could lie in the holographic principle. Such possibility is reinforced by the strong evidences that a quantum theory of spacetime must be holographic. Among such evidences, we have the recent results by Afshordi et al. which have shown that the universe has passed by a holographic phase at its early times [17]. Since it is also the realm where LQC contributions become necessary, it is required to find out a way to conciliate LQC with holography.

The applicability of the holographic principle to cosmology has been a subject of many discussions in the literature. In this way, following the pioneer work by Fischller and Suskind [18], a sort of scenarios has been proposed in order to establish the validity of the holographic principle in cosmological contexts, consisting in the so called Holographic Cosmology [18-25]. Moreover, the holographic hypothesis has a main role in braneworld cosmology via the application of the AdS/CFT formulation of the holographic principle to Randall-Sundrum braneworld scenario [26-32]. 
Among the versions of the holographic cosmology, Bak and Rey have argued that the holographic principle must be satisfied by the universe if one considers that its entropy must be associated with its apparent horizon [21]. (The definition of the apparent horizon of a Friedmann-Lemaitre-RobertsonWalker (FLRW) universe can be find, for example, in [33]). In this context, the validity of the first law of thermodynamics can be proved, which has made possible to derive the Friedmann equations of a FLRW universe [34] by the use of the Jacobson formalism [1].

By the way, the Jacobson formalism consists in one of the most important results in favor of the holographic hypothesis. In fact, the results by Jacobson have demonstrated that the Einstein's field equations can be derived from the proportionality between entropy and the horizon area, if the fundamental Clausius relation, $\delta Q=T d S$, connecting heat, temperature and entropy is valid for all the local Rindler causal horizon through each spacetime point, in a way that $\delta Q$ and $T$ will be interpreted, respectively, as the energy flux and Unruh temperature as seen by an accelerated observer just inside the horizon [1]. The results by Jacobson bring up an interesting consequence of the holographic principle: that the spacetime must have an atomic structure. In fact, the most important lesson which brings from such results is that the spacetime can be viewed as a gas of atoms with a related entropy given by the Bekenstein-Hawking formula, and the gravitational field equations are nothing, but equations of state describing this gas. Such an interpretation of spacetime was later reinforced by Padmanabhan, who linked the macroscopic description of spacetime, by Einstein equations, to microscopic degrees of freedom when assuming the principle of equipartition of energy [35]. A further extension of the Jacobson results to non-equilibrium situations has also been done [36]. The Jacobson's results have given rise to several works which have strengthened the thermodynamic interpretation of Einstein's equations. Actually, it has been shown that the susceptibility of gravitational fields to a thermodynamical behavior occurs not only in Einstein's gravity, but also in a wide assortment of theories. (For a review and a broad list of references see [37]).

One may think about to use the Jacobson's formalism in order to investigate the relationship between LQC and holography. In fact, such investigation has been done by Cai et al. [38]. In such work, the Jacobson's formalism has been used, where it has been taken into account a logarithmic quantum corrected Bekenstein-Hawking formula which arises in the context of LQG [39]. However, this attempt to obtain the LQC equations from Bekenstein-Hawking entropy, has led to quantum corrected Friedmann equations which give us a different scenario from LQC ones. Actually, the worse conclusion from such analysis is that a bounce does not occur anymore and the Big-Bang singularity is not resolved. It establishes a breakup between the description of the space- time behavior near the Big Bang and the way how its degrees of freedom are counted in the context of LQG. In this way, the important problem in reconciling the description of the universe provided by LQC with the thermodynamic evolution of the cosmos has been shown to be non trivial.

In the present work, we shall demonstrate that one can reconcile LQC and holography when it is considered that the universe entropy is given by the quantum corrected BekensteinHawking formula that arises in the context of Loop Quantum Black Holes (LQBHs). In order to do this, we shall show that LQC dynamical equations can be derived from LQBH's entropy-area relation, by the use of the Jacobson formalism. The present article is organized as follows: in Sect. 2, we shall review the basic aspects of LQBHs, in order to introduce the modified Bekenstein-Hawking formula that will be used throughout this paper; in Sect. 3 we shall review the formalism introduced by Cai et al. to derive the Friedmann equations from the Bekenstein-Hawking formula; in Sect. 4, we shall derive quantum corrected Friedmann equations from LQBHs' entropy-area relation; in Sect. 5, from the results of Sect. 4, we shall derive the LQC Friedmann equations; Sect. 6 is devoted to conclusions and perspectives. In this paper, unless otherwise stated, we shall use $\hbar=c=k_{B}=G=1$.

\section{Loop quantum black holes}

In this section we shall review the basic concepts of Loop Quantum Black Holes in order to introduce the quantum corrected Bekenstein-Hawking formula which will be used in order to derive the LQC dynamical equations.

In this way, a Loop Quantum Black Hole (LQBH), also called self-dual black hole, consists in a quantum corrected Schwarzschild black hole that appears from a simplified model of LQG by the use of semiclassical tools in the minisuperspace quantization approach [40-43].

The metric that describes the LQBH scenario is given by

$$
d s^{2}=-G(r) d t^{2}+F^{-1}(r) d r^{2}+H(r)\left(d \theta^{2}+\sin ^{2} \theta d \phi^{2}\right),
$$

where the functions above are defined as

$$
\begin{aligned}
& G(r)=\frac{\left(r-r_{+}\right)\left(r-r_{-}\right)\left(r+r_{*}\right)^{2}}{r^{4}+a_{0}^{2}}, \\
& F(r)=\frac{\left(r-r_{+}\right)\left(r-r_{-}\right) r^{4}}{\left(r+r_{*}\right)^{2}\left(r^{4}+a_{0}^{2}\right)} ; \quad H(r)=r^{2}+\frac{a_{0}^{2}}{r^{2}} .
\end{aligned}
$$

In this scenario, we have the presence of two horizons. The first one, localized at $r_{+}=2 m$, corresponds to an event horizon. The second one, localized at $r_{-}=2 m P^{2}$, corresponds to a Cauchy horizon. In addition, we have $r_{*}=\sqrt{r_{+} r_{-}}=2 m P$, where $P$ is the polymeric function defined by 
$P=\frac{\sqrt{1+\epsilon^{2}}-1}{\sqrt{1+\epsilon^{2}}+1}$.

In the definition above, $\epsilon=\gamma \delta_{b}$, being $\gamma$ the BarberoImmirzi parameter and $\delta_{b}$ the polymeric parameter which appear in the LQG quantization techniques. In particular, the polymeric parameter is used in order to define the length of the path along with the connection, used to define the holonomies in LQG, is integrated [40]. Moreover, $a_{0}=$ $A_{\min } / 8 \pi$, with $A_{\min }$ conceived as the minimal area value in LQG.

In the metric $2, r$ is only asymptotically the usual radial coordinate. It is because $g_{\theta \theta}$ is not just $r^{2}$. In this way, a more physical radial coordinate can be defined from the form of the function $H(r)$ in the metric:

$R=\sqrt{r^{2}+\frac{a_{0}^{2}}{r^{2}}}$

in the sense that the radial coordinate $R$ measures the proper circumferential distance. In addition, the relation of the parameter $m$ in the solution 1 with the ADM mass $M$ is given by $M=m(1+P)^{2}$.

An interesting feature of LQBHs is the property of selfduality. This property says that if one introduces the new coordinates $\tilde{r}=a_{0} / r$ and $\tilde{t}=t r_{*}^{2} / a_{0}$, with $\tilde{r}_{ \pm}=a_{0} / r_{\mp}$ the metric preserves its form. The dual radius is given by $r_{\text {dual }}=\tilde{r}=\sqrt{a_{0}}$ and corresponds to the minimal possible surface element. Moreover, since the Eq. 4 can be written as $R=\sqrt{r^{2}+\tilde{r}^{2}}$, it is clear that the solution contains another asymptotically flat Schwazschild region rather than a singularity in the limit $r \rightarrow 0$. This new region corresponds to a Planck-sized wormhole.

In addition, the Kretschmann invariant for this LQBH solution is given, for $r \approx 0$, by

$K=R_{\mu \nu \alpha \beta} R^{\mu \nu \alpha \beta}=\frac{3145728 \pi^{4} r^{6}}{a_{0}^{4} \gamma^{8} \delta^{8} m^{2}}$.

In a different way from the classical Schwarzschild scenario, the LQBH's Kretschmann invariant does not diverge when $r \rightarrow 0$. Such result points to the resolution of the singularity at $r=0$. As a matter of fact, in the quantum corrected LQBH scenario, an asymptotic flat region appears in the place of the black hole singularity, as has been shown in the LQBH Carter-Penrose diagram in Fig. 1.

The thermodynamic properties of LQBH's solution can be derived in the usual manner. In this way, the black hole temperature $T_{B H}$ is obtained by the calculation of the surface gravity $\kappa$, by $T_{B H}=\kappa / 2 \pi$, where

$\kappa^{2}=-g^{\mu \nu} g_{\rho \sigma} \nabla_{\mu} \chi^{\rho} \nabla_{\nu} \chi^{\sigma}=-\frac{1}{2} g^{\mu \nu} g_{\rho \sigma} \Gamma_{\mu 0}^{\rho} \Gamma_{\nu 0}^{\sigma}$.

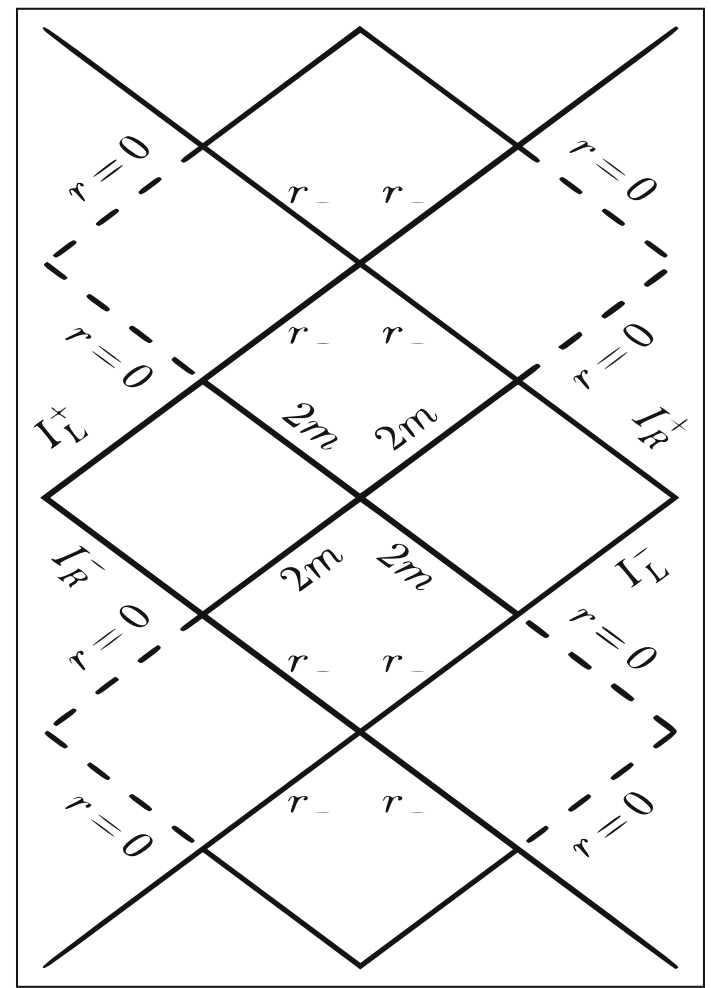

Fig. 1 Carter-Penrose diagram for the self-dual black hole solution. The LQBH scenario is endowed with two asymptotic flat regions, one placed at the infinity and the other one near the origin. The second region can not be reached by any observer in a finite time

In the equation above, $\chi^{\mu}=(1,0,0,0)$ consists in a timelike Killing vector. On the other hand, $\Gamma_{\sigma \rho}^{\mu}$ give us the connections coefficients.

By connecting with the metric, one obtains the LQBH's temperature

$T_{H}=\frac{(2 m)^{3}\left(1-P^{2}\right)}{4 \pi\left[(2 m)^{4}+a_{0}^{2}\right]}$.

It is easy to see that one can recover the usual Hawking temperature in the limit of large masses. However, differently from the Hawking case, the temperature 7 goes to zero for $m \rightarrow 0$.

The black hole's entropy can be found out by making use of the thermodynamical relation $S_{B H}=\int d m / T(m)$,

$S=\frac{4 \pi}{\left(1-P^{2}\right)}\left[\frac{16 m^{4}-a_{0}^{2}}{16 m^{2}}\right]$.

From the expression 8 , one can obtain an expression for the black hole entropy in terms of its area [43]

$S= \pm \frac{\sqrt{A^{2}-A_{\min }^{2}}}{4 \sigma}$ 
where $\sigma=1-P^{2}$, and we have chosen the possible additional constant to be zero. In addition, $A=4 \pi R^{2}$, with $R$ defined by the Eq. 4 . We have that, $\mathrm{S}$ has a positive value for $m>\sqrt{a_{0}} / 2$ and becomes negative otherwise. The two possible situations for the signal of the loop black hole entropy are related to the double physical possibility that arises from LQBH's structure, depending on the localization of the event horizon ( outside or inside of the wormhole throat) [42].

Another way to obtain the thermodynamic properties of LQBH has been pointed in [44], where the Hamilton-Jacobi version of the tunneling formalism has been used. In this context, back-reaction effects have been included. Moreover, the LQBH solution can be extended to scenarios of black holes with charge and angular momentum [45]. The information loss problem has also been discussed in the LQBH's framework. As an important result, the problem of information loss by black holes can be relieved in this scenario $[44,46]$.

\section{Friedmann equations from thermodynamics}

In this section, we shall review the method introduced by Cai and Kim [34] and Cai et al. [38] in order to derive the Friedmann equations from the Bekenstein-Hawking formula, by considering that the holographic bound is satisfied by the universe in some regime. Such method is based on the results by Jacobson that demonstrated the equivalence between the Einstein's gravitational equations and thermodynamics [1].

In this sense, in order to have the holographic bound fulfilled by the universe, we shall consider the evolution of a universe region whose holographic boundary corresponds to the cosmological apparent horizon. The issue of the correct choice of the cosmological holographic boundary has been a point of some discussion in the literature. In this way, such a boundary has been considered as having the size of the Hubble horizon in $[19,25]$, the size of the apparent horizon in [21], and the size of the particle horizon in [24]. However, in order to get a thermodynamic description of the universe evolution based on the Jacobson formalism, it has been shown that the choice of the cosmological apparent horizon as the holographic boundary $[21,34]$ is more convenient. Such conclusion comes from that fact that, at the apparent horizon, the Friedmann equations have been shown to be equivalent to the first law of thermodynamics [47-49]. It occurs not only in Einstein's gravity, but in other scenarios such as braneworld models [50-53], Horava-Lifshitz gravity [54], Lovelock gravity [47,55] and $f(R)$ gravity [56]. Moreover, it has been shown that the obedience to the generalized second law of thermodynamics is fulfilled in the scenario of an accelerating expanding universe when one identifies the cosmological holographic boundary as the universe apparent horizon [57-59]. On the base of such facts, it can be argued that the apparent horizon must be considered as the phys- ical horizon in dealing with thermodynamics issues, in the context of a universe with any curvature.

Following the procedure developed in $[34,38]$, in order to obtain the LQC Friedmann equations, we have that the FLRW universe is described by the following metric

$$
\begin{aligned}
d s^{2} & =-d t^{2}+a(t)^{2}\left(\frac{d r^{2}}{1-k r^{2}}+r^{2} d \Omega_{2}^{2}\right) \\
& =h_{a b} d x^{a} d x^{b}+\tilde{r}^{2} d \Omega_{2}^{2},
\end{aligned}
$$

where $h_{a b}=\operatorname{diag}\left(-1, a^{2} /\left(1-k r^{2}\right)\right)$ and $\tilde{r}=a(t) r$. Moreover, the radius of the apparent horizon is given by

$\tilde{r}_{A}=\frac{1}{\sqrt{H^{2}+k / a^{2}}}$,

Now, let us consider that the energy-momentum tensor $T_{\mu \nu}$ related with the matter in universe possess the form of the one for a perfect fluid:

$T_{\mu \nu}=(\rho+p) U_{\mu} U_{\nu}+p g_{\mu \nu}$.

From the energy conservation law, comes the continuity equation

$\dot{\rho}+3 H(\rho+p)=0$.

In this point, let us introduce the work density $W$ and the energy-supply vector $\psi_{a}$

$W=-\frac{1}{2} T^{a b} h_{a b}$

and

$\psi_{a}=T_{a}^{b} \partial_{b} \tilde{r}+W \partial_{a} \tilde{r}$.

We shall have, in our case

$W=\frac{1}{2}(\rho-p)$

and

$\psi_{a}=-\frac{1}{2}(\rho+p) H \tilde{r} d t+\frac{1}{2}(\rho+p) a d r$.

From the expressions above, we can compute the amount of energy going through the apparent horizon during the time interval $d t$ as [34]

$\delta Q=-A \psi=A(\rho+p) H \tilde{r}_{A} d t$

where $A=4 \pi \tilde{r}_{A}^{2}$. 
The gravitational equations are obtained by the use the Clausius relation $\delta Q=T d S$, where the universe entropy is conjectured to be given by the Bekenstein-Hawking formula

$S=\frac{A}{4}$

On the other hand, the temperature associated with the universe apparent horizon is given by

$T=\frac{1}{2 \pi \tilde{r}_{A}}$,

which was obtained in the reference $[48,49]$ through tunneling methods.

From the equations above, we obtain

$\dot{H}-\frac{k}{a^{2}}=4 \pi(\rho+p)$.

In order to obtain the Friedmann equation above we have used the relation

$\dot{\tilde{r}}_{A}=-H \tilde{r}_{A}^{3}\left(\dot{H}-\frac{k}{a^{2}}\right)$.

Now, using the continuity Eq. 13, we can find

$$
\begin{aligned}
\frac{8 \pi}{3} d \rho & =d\left(H^{2}+k / a^{2}\right) \\
& =\frac{4 \pi}{A^{2}} d A,
\end{aligned}
$$

where we have used the fact that $H^{2}+\frac{k}{a^{2}}=\frac{4 \pi}{A}$.

The integration of the Eq. 23 give us

$H^{2}+\frac{k}{a^{2}}=\frac{8 \pi}{3} \rho$,

which consists in the first Friedmann equation.

Now, by the time differentiation of the equation above, and the use of the continuity Eq. 13 we find

$\dot{H}-\frac{k}{a^{2}}=4 \pi(\rho+p)$,

which constitutes in the second Friedmann equation, i.e., the Raychaudhuri equation.

In this way, the complete dynamics of a FLRW universe can be find out from thermodynamics. Such results have been applied in order to study a sort of problems in cosmology, in particular, such related with the thermodynamic evolution of the universe. In this way, the validity of the Generalized Second law of thermodynamics has been investigated in this context $[60,61]$. Moreover, such formalism has been used to study the relation between gravity and thermodynamics in the context of extended theories of gravity such scalartensor gravity and $\mathrm{f}(\mathrm{R})$ gravity [62], braneworld scenarios [50,51,53,63], viscous cosmology [64], and Horava-Lifshitz gravity [65].

\section{Quantum corrected Friedmann equations from LQBHs}

In this section, we shall obtain quantum corrected Friedmann equations for the evolution of the universe, by considering that the holographic bound is satisfied near the Big Bang/Big Crunch singularity, where we shall assume that the entropy associated with the universe apparent horizon is related with its area by the modified entropy-area relation 9 .

In this way, upon the same considerations of the last section, following the procedure developed in [34,38], in order to obtain the gravitational equations, we have that by the use of the Clausius relation $\delta Q=T d S$, and the LQBH entropyarea relation 9 we can reach

$\dot{H}-\frac{k}{a^{2}}=\mp 4 \pi \sigma \frac{\sqrt{A^{2}-A_{\text {min }}^{2}}}{A}(\rho+p)$.

Now, using the continuity Eq. 13, we can find

$$
\begin{aligned}
\frac{8 \pi}{3} d \rho & = \pm \frac{1}{\sigma} \frac{A}{\sqrt{A^{2}-A_{\text {min }}^{2}}} d\left(H^{2}+k / a^{2}\right) \\
& =\mp \frac{1}{\sigma} \frac{4 \pi}{A \sqrt{A^{2}-A_{\text {min }}^{2}}} d A,
\end{aligned}
$$

where, again we have used the fact that $H^{2}+\frac{k}{a^{2}}=\frac{4 \pi}{A}$.

The integration of the Eq. 27 give us

$\Theta= \pm\left[\frac{2 A_{\min }}{3} \sigma \rho-\alpha\right]=\arccos \left(A_{\min } / A\right)$,

where we must have $-\pi / 2 \leq \Theta \leq \pi / 2$, since $A_{\min } / A \geq 0$.

The Eq. 28 give us the following Friedmann equation:

$H^{2}+\frac{k}{a^{2}}=\frac{1}{\gamma \sqrt{3}} \cos (\Theta)$,

where we have used $A_{\min }=4 \pi \gamma \sqrt{3}[66]$.

The Raychaudhuri equation reads

$\dot{H}-\frac{k}{a^{2}}=\frac{1}{\gamma \sqrt{3}} \sigma(\rho+p) \sin (\Theta)$.

The Eqs. 29 and 30 consist in the quantum versions of the Friedmann equations. As we can see, the quantum corrections present in these equations, inherited from the LQBH's entropy-area relation, imply in a quantum effective density term which is a harmonic function of the classical density. A 
very important consequence of this result is that the quantum corrected Friedmann equations bring us a scenario where the Big Bang initial singularity does not exist anymore, but is replaced by a bounce at a point where the universe density gets a critical value, as occurs in LQC. In the Eq. 29, the phase constant $\alpha$ will be given by the initial conditions of the universe and must be appropriately chosen in order to ensure that the effective density term is definite positive, in concordance with the Eq. 28 and the comment below it.

\section{Relation with usual semiclassical LQC}

In this point, we shall address how the quantum corrected Friedmann equations found out in the last section can be related to the usual semiclassical LQC equations. In order to do this, let us expand the Eq. 29 as

$$
\begin{aligned}
H^{2}+\frac{k}{a^{2}}= & \frac{1}{\gamma \sqrt{3}} \cos (\alpha)+\frac{8 \pi}{3} \sigma \sin (\alpha) \rho \\
& -\frac{32 \pi^{2}}{9} \gamma \sigma^{2} \sqrt{3} \rho^{2} \cos (\alpha),
\end{aligned}
$$

where we have disconsidered the terms that depend on quantum corrections of order $\mathcal{O}\left(\geq A_{\text {min }}^{2}\right)$, as have been done in the usual semiclassical LQC [67].

The equation above can be written in the form

$H^{2}+\frac{k}{a^{2}}=\frac{8 \pi}{3} \rho_{t o t}\left(1-\frac{\rho_{t o t}}{\rho_{c}}\right)$,

where $\rho_{\text {tot }}=\rho+\frac{\Lambda}{8 \pi}$, with $\Lambda$ as a cosmological constant.

The Raychaudhuri equation can also be obtained from the time derivative of the Eq. 32 which, by the use of the continuity Eq. 13, give us

$\dot{H}-\frac{k}{a^{2}}=-4 \pi\left(\rho_{t o t}+p_{t o t}\right)\left(1-\frac{2 \rho_{t o t}}{\rho_{c}}\right)$,

where $p_{\text {tot }}=p-\frac{\Lambda}{8 \pi}$. In this way, one could obtain the complete LQC semiclassical dynamics from an holographic prescription by the use of the LQBH entropy-area relation.

However, there are still some points to address. The first point is the role of the cosmological constant which appears, in our approach, from the quantum gravity corrections to the Bekenstein-Hawking formula. The second point consists in how to conciliate the universe critical density found out in our treatment to that given by usual LQC.

In order to discuss such points, we have that from the Eqs. 31 and 32, we must have $\rho_{c}=\frac{\sqrt{3}}{4 \pi \gamma \sigma^{2} \cos (\alpha)}$

$1-\frac{2 \tilde{\Lambda}}{\rho_{c}}=\sigma \sin (\alpha)$,

$\tilde{\Lambda}\left(1-\frac{\tilde{\Lambda}}{\rho_{c}}\right)=\frac{3 \cos (\alpha)}{8 \pi \gamma \sqrt{3}}$,

where $\tilde{\Lambda}=\frac{\Lambda}{8 \pi}$.

Moreover, from the Eqs. 34, 35 and 36, we shall find

$\tilde{\Lambda}_{ \pm}=\frac{\rho_{c}}{2}\left(1 \pm \sqrt{1-4 \frac{\xi_{ \pm}}{\rho_{c}}}\right)$

where

$\xi_{ \pm}=\frac{\rho_{c}}{4}\left(1 \pm \sqrt{1-\frac{3}{4 \pi^{2} \gamma^{2} \rho_{c}^{2}}}\right)$

We also obtain

$\sigma^{2}=\frac{3}{32 \pi^{2} \gamma^{2} \rho_{c}}\left[\tilde{\Lambda}\left(1-\frac{\tilde{\Lambda}}{\rho_{c}}\right)\right]^{-1}$,

and

$\cos (\alpha)^{2}=\frac{64 \pi^{2} \gamma^{2}}{3}\left[\tilde{\Lambda}\left(1-\frac{\tilde{\Lambda}}{\rho_{c}}\right)\right]^{2}$.

In the Eq. 38, $\xi_{-}$is the only consistent choice in order to have a real valued solution to 37 . On the other hand, in the Eq. $37, \tilde{\Lambda}_{-}$is the only choice consistent with the agreement between the Eq. 40 and the condition that $-1 \leq \cos (\alpha) \leq 1$. In this way, we obtain:

$\Lambda=8 \pi \tilde{\Lambda}=4 \pi \rho_{c}\left[1-\left(1-\frac{3}{4 \pi^{2} \gamma^{2} \rho_{c}^{2}}\right)^{\frac{1}{4}}\right]$.

Consequently, the value of the cosmological constant depends on the initial conditions of the universe, particularly on the value of the universe critical density at the bounce. In the limit where $\rho_{c} \gg 1, \Lambda \sim \frac{3}{4 \pi \gamma^{2} \rho_{c}}$, in a way that, in the infrared limit $\left(\rho_{c} \rightarrow \infty\right), \Lambda \rightarrow 0$.

By comparing the appearance of a cosmological constant in our treatment with usual LQC, it has been demonstrated that LQC fits the situation where a cosmological constant $\Lambda$ is present, for both the cases where $\Lambda$ is positive $[68,69]$ or negative [70]. However, LQC does not offer any theoretical result for the value of the cosmological constant, within the sense that it does not arise as the result of a more fundamental calculation [71-73]. Instead, the point of view in LQC 
(and, more generally, in LQG) has been that the cosmological constant consists in a constant of nature, in the same sense of Newton's gravitational constant or Planck's constant, or the electron charge. From this standpoint, $\Lambda$ should be measured through some experiments and/or observations. As a consequence, LQC does not address what is often called the " "cosmological constant problem" which asks for an explanation from fundamental physics of why the observed value of $\Lambda$ is so small compared to that provided by the Standard Model, which predicts $\Lambda$ would be Planckian. Such persistent problem is considered as one of the most puzzling in physics. (For a recent review about the cosmological constant problem, see [74]).

The results found out in the present article, on the other hand, tie the cosmological constant to the universe density at the bounce. In this way, concerning to the value of the universe critical density, we have that, in the context of LQC, it is given by $[67,75-80]$

$$
\rho_{c}=\frac{3}{8 \pi \gamma^{2} \Delta},
$$

where $\Delta$ is an area gap.

Usually one assumes that the area gap above is given by the LQG one, $\Delta_{L Q G}=4 \pi \gamma \sqrt{3}$, and calculates the value of the universe critical density to be, in Planck units, $\rho_{c} \approx 0.41$ $[79,80]$. The same numerical result for $\rho_{c}$ is obtained when we have the presence of a cosmological constant [68-70]. However, such assumption can not be taken into account in the present formalism since, in such a case, the cosmological constant given by the Eq. 41 would be not real valued. In fact in order to have a real valued cosmological constant in the Eq. 41, one must have

$\rho_{c} \geq \frac{\sqrt{3}}{2 \pi \gamma} \approx 1.16$

It could be a bone of contention between our treatment and the usual LQC.

However, it has been pointed that the choice of the full LQG area gap in order to calculate $\rho_{c}$ is naive and lacks further physical justification. Consequently, other values for the universe critical density could be conceived. In fact, from the arguments presented in [81-83], the value of $\rho_{c}$ would be fixed by observations. For a more general expression for $\rho_{c}$, see [84].

If it is this case, the results of the present work could give us a way to fix the value of $\rho_{c}$ by the use of the observational results about $\Lambda$. In this way, one must have that the energy scale of the bounce would be super Planckian, since in order to have a concordance with the observed value of the cosmological constant one must have $\rho_{c} \sim 10^{120}$.

\section{Summary and conclusions}

Loop quantum gravity is a propose to the description of spacetime behavior in situations where its atomic characteristic arises. Among these situations, the nature of our universe near the Big Bang singularity is described by LQC. Near the Big Bang, LQC faces some important questions about the thermodynamical evolution of the universe for what the holographic principle must be fundamental. Among such questions, the origin of the universe entropy, and the arrow of time [11].

However, an investigation of LQC under the holographic point of view was still lacking. In this way, in the present work, we have shown a manner to obtain the LQC semiclassical equations from the holographic principle. In order to do this, we have considered that the entropy of the universe is given by the LQBH's entropy-area relation, and the holographic boundary is chosen as the universe apparent horizon in order to have the validity of the first law of thermodynamics. Based on such assumptions, by the use of the Jacobson formalism [1], adapted to cosmological scenarios by Cai et al. [34], the dynamical LQC equations can be obtained.

The compatibility with the results of standard LQC, might suggest that the black hole's entropy counting performed in loop black hole's scenario may be on a more solid footing, in cosmological contexts, than other counting procedures such as those given in other approaches like [39]. Since the Eq. 29 is an equation of state for the cosmological evolution of spacetime, semiclassical LQC would appear as a thermodynamic effect whose origin would lies in the atomic structure of spacetime described by LQG.

Among our results, a positive cosmological constant has been obtained. The value of the cosmological constant depends on the universe initial conditions, specially it depends on the choice of the universe critical density at the bounce. In order to have a real valued cosmological constant, the naive assumption that the universe critical density is determined by the LQG area gap can not be considered in the present formalism. On the other hand, there are some suggestions that $\rho_{c}$ should be fixed by observations. In this case, our results gives a way to fix the value of $\rho_{c}$ by connecting it with the cosmological constant observed value. In this way $\rho_{c} \sim 10^{120}$. The problem of why the cosmological constant has a very small value in our universe remains open.

The results found out in this paper can pave the way for a large sort of investigation of important issues about the thermodynamical behavior of our universe as described by LQC. Besides the aforementioned problems of the origin of universe entropy and the arrow of time, non-equilibrium regimes can be also investigated in this context.

Acknowledgements The author would like to thank the anonymous referee by the useful discussions and comments about the paper. 
Open Access This article is distributed under the terms of the Creative Commons Attribution 4.0 International License (http://creativecomm ons.org/licenses/by/4.0/), which permits unrestricted use, distribution, and reproduction in any medium, provided you give appropriate credit to the original author(s) and the source, provide a link to the Creative Commons license, and indicate if changes were made.

Funded by SCOAP ${ }^{3}$.

\section{References}

1. T. Jacobson, Phys. Rev. Lett. 75, 1260 (1995)

2. C. Rovelli, Quantum Gravity (Cambridge University Press, Cambridge, 2004)

3. A. Ashtekar, J. Lewandowski, Class. Quant. Grav. 21, R53 (2004). arXiv:gr-qc/0404018

4. T. Thiemann, Lect. Notes Phys. 721, 185 (2007). arXiv:hep-th/0608210

5. T. Thiemann. arXiv:gr-qc/0110034

6. T. Thiemann, Lect. Notes Phys. 631, 41 (2003). arXiv:gr-qc/0210094

7. M. Bojowald, Phys. Rev. Lett. 86, 5227 (2001)

8. A. Barrau, T. Cailleteau, J. Grain, J. Mielczarek, Class. Quant. Grav. 31, 053001 (2014)

9. A. Ashtekar, A. Barrau. arXiv:1504.07559 [gr-qc]

10. M. Bojowald, Nat. Phys. 3(8), 523 (2007)

11. M. Bojowald, R. Tavakol, Phys. Rev. D 78, 023515 (2008)

12. J.D. Bekenstein, Phys. Rev. D 7, 2333 (1973)

13. G. 't Hooft, Salamfest, 0284-296 (1993). arXiv:gr-qc/9310026

14. L. Susskind, J. Math. Phys. 36, 6377 (1995)

15. R. Bousso, Rev. Mod. Phys. 74, 825 (2002)

16. L. Susskind, Nat. Phys. 2(10), 665 (2006)

17. N. Afshordi, C. Coriano, L. Delle Rose, E. Gould, K. Skenderis, Phys. Rev. Lett. 118(4), 041301 (2017). https://doi.org/10.1103/ PhysRevLett.118.041301. arXiv:1607.04878 [astro-ph.CO]

18. W. Fischler, L. Susskind. arXiv:hep-th/9806039

19. G. Veneziano, Phys. Lett. B 454, 22 (1999). https://doi.org/10. 1016/S0370-2693(99)00267-1. arXiv:hep-th/9902126

20. R. Easther, D.A. Lowe, Phys. Rev. Lett. 82, 4967 (1999). https:// doi.org/10.1103/PhysRevLett.82.4967. arXiv:hep-th/9902088

21. D. Bak, S.J. Rey, Class. Quant. Grav. 17, L83 (2000). https://doi. org/10.1088/0264-9381/17/15/101. arXiv:hep-th/9902173

22. R. Bousso, JHEP 9907, 004 (1999). https://doi.org/10.1088/ 1126-6708/1999/07/004. arXiv:hep-th/9905177

23. R. Bousso, JHEP 9906, 028 (1999). https://doi.org/10.1088/ 1126-6708/1999/06/028. arXiv:hep-th/9906022

24. T. Banks, W. Fischler. arXiv:hep-th/0111142

25. N. Kaloper, A.D. Linde, Phys. Rev. D 60, 103509 (1999). https:// doi.org/10.1103/PhysRevD.60.103509. arXiv:hep-th/9904120

26. L. Randall, R. Sundrum, Phys. Rev. Lett. 83, 4690 (1999). https:// doi.org/10.1103/PhysRevLett.83.4690. arXiv:hep-th/9906064

27. S.S. Gubser, Phys. Rev. D 63, 084017 (2001). https://doi.org/10. 1103/PhysRevD.63.084017. arXiv:hep-th/9912001

28. I. Savonije, E.P. Verlinde, Phys. Lett. B 507, 305 (2001). https:// doi.org/10.1016/S0370-2693(01)00467-1. arXiv:hep-th/0102042

29. S. Mukohyama, Phys. Lett. B 473, 241 (2000). https://doi.org/10. 1016/S0370-2693(99)01505-1. arXiv:hep-th/9911165

30. E.E. Flanagan, S.H.H. Tye, I. Wasserman, Phys. Rev. D 62, 044039 (2000). https://doi.org/10.1103/PhysRevD.62.044039. arXiv:hep-ph/9910498

31. P. Binetruy, C. Deffayet, U. Ellwanger, D. Langlois, Phys. Lett. B 477, 285 (2000). https://doi.org/10.1016/ S0370-2693(00)00204-5. arXiv:hep-th/9910219

32. D. Ida, JHEP 0009, 014 (2000). https://doi.org/10.1088/ 1126-6708/2000/09/014. arXiv:gr-qc/9912002
33. V. Faraoni, Lect. Notes Phys. 907, 1 (2015). https://doi.org/10. 1007/978-3-319-19240-6

34. R.G. Cai, S.P. Kim, JHEP 0502, 050 (2005)

35. T. Padmanabhan, Phys. Rev. D 81, 124040 (2010)

36. C. Eling, R. Guedens, T. Jacobson, Phys. Rev. Lett. 96, 121301 (2006). arXiv:gr-qc/0602001

37. T. Padmanabhan, Rept. Prog. Phys. 73, 046901 (2010)

38. R.G. Cai, L.M. Cao, Y.P. Hu, JHEP 0808, 090 (2008)

39. K.A. Meissner, Class. Quant. Grav. 21, 5245 (2004)

40. L. Modesto, Int. J. Theor. Phys. 49, 1649 (2010). https://doi.org/ 10.1007/s10773-010-0346-x. arXiv:0811.2196 [gr-qc]

41. L. Modesto, I. Premont-Schwarz, Phys. Rev. D 80, 064041 (2009)

42. B. Carr, L. Modesto, I. Premont-Schwarz. arXiv:1107.0708 [gr-qc]

43. S. Hossenfelder, L. Modesto, I. Premont-Schwarz. arXiv:1202.0412 [gr-qc]

44. C.A.S. Silva, F.A. Brito, Phys. Lett. B 725(45), 456 (2013)

45. F. Caravelli, L. Modesto, Class. Quant. Grav. 27, 245022 (2010)

46. E. Alesci, L. Modesto, Gen. Rel. Grav. 46, 1656 (2014)

47. Y. Gong, A. Wang, Phys. Rev. Lett. 99, 211301 (2007). https://doi. org/10.1103/PhysRevLett.99.211301. arXiv:0704.0793 [hep-th]

48. R.G. Cai, L.M. Cao, Phys. Rev. D 75, 064008 (2007). https://doi. org/10.1103/PhysRevD.75.064008. arXiv:gr-qc/0611071

49. R.G. Cai, L.M. Cao, Y.P. Hu, Class. Quant. Grav. 26, 155018 (2009)

50. R.G. Cai, L.M. Cao, Nucl. Phys. B 785, 135 (2007). https://doi. org/10.1016/j.nuclphysb.2007.06.016. arXiv:hep-th/0612144

51. R.G. Cai, Prog. Theor. Phys. Suppl. 172, 100 (2008). https://doi. org/10.1143/PTPS.172.100. arXiv:0712.2142 [hep-th]

52. A. Sheykhi, B. Wang, R.G. Cai, Nucl. Phys. B 779, 1 (2007). https:// doi.org/10.1016/j.nuclphysb.2007.04.028. arXiv:hep-th/0701198

53. A. Sheykhi, B. Wang, R.G. Cai, Phys. Rev. D 76, 023515 (2007). https://doi.org/10.1103/PhysRevD.76.023515. arXiv:hep-th/0701261

54. R.G. Cai, L.M. Cao, N. Ohta, Phys. Lett. B 679, 504 (2009). https://doi.org/10.1016/j.physletb.2009.07.075. arXiv:0905.0751 [hep-th]

55. R.G. Cai, L.M. Cao, Y.P. Hu, S.P. Kim, Phys. Rev. D 78, 124012 (2008). https://doi.org/10.1103/PhysRevD.78.124012. arXiv:0810.2610 [hep-th]

56. Y. Zhang, Y. Gong, Z.H. Zhu, Int. J. Mod. Phys. D 21, 1250034 (2012). https://doi.org/10.1142/S0218271812500344

57. B. Wang, Y. Gong, E. Abdalla, Phys. Rev. D 74, 083520 (2006). https://doi.org/10.1103/PhysRevD.74.083520. arXiv:gr-qc/0511051

58. J. Zhou, B. Wang, Y. Gong, E. Abdalla, Phys. Lett. B 652, 86 (2007). https://doi.org/10.1016/j.physletb.2007.06.067. arXiv:0705.1264 [gr-qc]

59. A. Sheykhi, Class. Quant. Grav. 27, 025007 (2010). https://doi.org/ 10.1088/0264-9381/27/2/025007. arXiv:0910.0510 [hep-th]

60. S.F. Wu, B. Wang, G.H. Yang, P.M. Zhang, Class. Quant. Grav. 25, 235018 (2008). https://doi.org/10.1088/0264-9381/25/ 23/235018. arXiv:0801.2688 [hep-th]

61. N. Radicella, D. Pavon, Phys. Lett. B 691, 121 (2010). https://doi. org/10.1016/j.physletb.2010.06.019. arXiv:1006.3745 [gr-qc]

62. M. Akbar, R.G. Cai, Phys. Lett. B 635, 7 (2006). https://doi.org/ 10.1016/j.physletb.2006.02.035. arXiv:hep-th/0602156

63. X.H. Ge, Phys. Lett. B 651, 49 (2007). https://doi.org/10.1016/j. physletb.2007.05.055. arXiv:hep-th/0703253

64. M. Akbar, Chin. Phys. Lett. 25, 4199 (2008). https://doi.org/10. 1088/0256-307X/25/12/004. arXiv:0808.0169 [gr-qc]

65. A. Sheykhi, Phys. Rev. D 87(2), 024022 (2013). https://doi.org/10. 1103/PhysRevD.87.024022. arXiv:1301.3776 [hep-th]

66. A. Perez. arXiv:gr-qc/0409061

67. V. Taveras, Phys. Rev. D 78, 064072 (2008). https://doi.org/10. 1103/PhysRevD.78.064072. arXiv:0807.3325 [gr-qc] 
68. W. Kaminski, T. Pawlowski, Phys. Rev. D 81, 024014 (2010). https://doi.org/10.1103/PhysRevD.81.024014. arXiv:0912.0162 [gr-qc]

69. T. Pawlowski, A. Ashtekar, Phys. Rev. D 85, 064001 (2012). https:// doi.org/10.1103/PhysRevD.85.064001. arXiv:1112.0360 [gr-qc]

70. E. Bentivegna, T. Pawlowski, Phys. Rev. D 77, 124025 (2008). https://doi.org/10.1103/PhysRevD.77.124025. arXiv:0803.4446 [gr-qc]

71. E. Bianchi, C. Rovelli. arXiv:1002.3966 [astro-ph.CO]

72. E. Bianchi, C. Rovelli, R. Kolb, Nature, 466, 321 (2010)

73. E. Wilson-Ewing, Comptes Rendus Phys. 18, 207 (2017). https:// doi.org/10.1016/j.crhy.2017.02.004. arXiv:1612.04551 [gr-qc]

74. A. Padilla. arXiv: 1502.05296 [hep-th]

75. A. Ashtekar, T. Pawlowski, P. Singh, Phys. Rev. D 73, 124038 (2006). https://doi.org/10.1103/PhysRevD.73.124038. arXiv:gr-qc/0604013

76. A. Ashtekar, T. Pawlowski, P. Singh, Phys. Rev. D 74, 084003 (2006). https://doi.org/10.1103/PhysRevD.74.084003. arXiv:gr-qc/0607039

77. A. Ashtekar, T. Pawlowski, P. Singh, Phys. Rev. Lett. 96, 141301 (2006). https://doi.org/10.1103/PhysRevLett.96.141301. arXiv:gr-qc/0602086
78. A. Ashtekar, A. Corichi, P. Singh, Phys. Rev. D 77, 024046 (2008). https://doi.org/10.1103/PhysRevD.77.024046. arXiv:0710.3565 [gr-qc]

79. D.W. Chiou, K. Vandersloot, Phys. Rev. D 76, 084015 (2007). https://doi.org/10.1103/PhysRevD.76.084015. arXiv:0707.2548 [gr-qc]

80. A. Ashtekar, Gen. Rel. Grav. 41, 707 (2009). https://doi.org/10. 1007/s10714-009-0763-4. arXiv:0812.0177 [gr-qc]

81. P. Malkiewicz, W. Piechocki, Phys. Rev. D 80, 063506 (2009). https://doi.org/10.1103/PhysRevD.80.063506. arXiv:0903.4352 [gr-qc]

82. M. Bojowald, Class. Quant. Grav. 26, 075020 (2009). https://doi. org/10.1088/0264-9381/26/7/075020. arXiv:0811.4129 [gr-qc]

83. P. Dzierzak, J. Jezierski, P. Malkiewicz, W. Piechocki, Acta Phys. Polon. B 41, 717 (2010). arXiv:0810.3172 [gr-qc]

84. M. Bojowald, Gen. Rel. Grav. 40, 2659 (2008). https://doi.org/10. 1007/s10714-008-0645-1. arXiv:0801.4001 [gr-qc] 